\title{
Myelolipomatous Adrenal Adenoma and Adrenal Scintigraphy: A Case Presentation and Review of Literature
}

\author{
Sangeeta Chandramahantia, d, Prasanna Santhanam ${ }^{b}$ David Taieb $^{c}$
}

\begin{abstract}
Adrenal myelolipomas are usually benign and non-functioning masses. On the other hand, adrenal myelolipomatous adenoma that may mimic adrenal myelolipoma on conventional imaging may be a rare cause of subclinical Cushing's disease. Adrenal scintigraphy (with ${ }^{131}$ I-6beta-iodomethyl-19-norcholesterol) can be used to demonstrate increased uptake in a functioning adrenal myelolipomatous adenoma to distinguish it from an adrenal myelolipoma, when the source of the excess hormone secretion after biochemical evaluation is unclear. Treating physicians should be familiar with the occasional use of this imaging modality that can help distinguish functional adrenal tumors from non-functioning ones when conventional imaging fails to do so. A 66-year-old female was found to have an incidental 6.5 $\mathrm{cm}$ right adrenal mass during a routine abdominal ultrasound. Complete biochemical evaluation was suggestive of subclinical Cushing's syndrome. Imaging studies were consistent with adrenal myelolipoma. Adrenal scintigraphy showed a unilateral increased uptake at the site of the adrenal mass. She successfully underwent laparoscopic adrenalectomy of the $6.5 \mathrm{~cm}$ mass and she had significant clinical improvement. Myelolipomatous adrenal adenoma (that may mimic adrenal myelolipoma on conventional imaging) may be a rare cause of subclinical Cushing's, and adrenal scintigraphy may be a useful imaging technique that may help in localizing the lesion, which can then be treated surgically.
\end{abstract}

Keywords: Adrenal cortex neoplasms; 19-Iodocholesterol; Myelolipoma

\footnotetext{
Manuscript accepted for publication December 02, 2014

aSection of Endocrinology, Internal Medicine, Joan C Edwards School of Medicine, Marshall University, 1249 15th Street, Suite 300, Huntington, WV 25701, USA

bDepartment of Internal Medicine, Joan C Edwards School of Medicine, Marshall University, 1249 15th Street, Suite 300, Huntington, WV 25701, USA

'Department of Nuclear Medicine, La Timone University Hospital, Aix-Marseille University, CERIMED, France

${ }^{\mathrm{d} C}$ Corresponding Author: Sangeeta Chandramahanti, Section of Endocrinology, Internal Medicine, Joan C Edwards School of Medicine, Marshall University, 1249 15th Street, Suite 300, Huntington, WV 25701, USA.

Email: chandramahan@live.marshall.edu
}

doi: http://dx.doi.org/10.14740/jem254w

\section{Introduction}

Adrenal scintigraphy (with ${ }^{131}$ I-6beta-iodomethyl-19-norcholesterol) is seldom used in clinical practice in the US. However, there are certain circumstances where it might be helpful especially when there is a suspicion for subclinical Cushing's. We present a case and review of literature about the use of adrenal scintigraphy.

\section{Case Report}

A 66-year-old woman presented with an adrenal incidentaloma found during a regular abdominal ultrasound. Her medical history included severe obesity (BMI 38.6), type 2 diabetes treated with insulin, resistant arterial hypertension, coronary artery disease (history of placement of coronary stents) and chronic congestive heart failure. Complete hormonal workup found subclinical (ACTH independent) Cushing's syndrome: labs showed an elevated $24 \mathrm{~h}$ urine free cortisol $(85 \mathrm{nmol} / 24$ h), suppressed ACTH $(<1 \mathrm{pmol} / \mathrm{L})$ with an absence of cortisol suppression $(113 \mathrm{nmol} / \mathrm{L}, \mathrm{N}<50)$ after $2 \mathrm{mg}$ dexamethasone suppression test with DHEA of $0.2 \mu \mathrm{mol} / \mathrm{L}$ (normal range 0.26 - 6.68).

Abdominal CT and MRI scan showed a $6.5 \mathrm{~cm}$ right adrenal mass containing soft tissue, calcifications and macroscopic fat with no loss of signal intensity in the out-of-phase image(s) in the MRI (Fig. 1). A diagnosis of myelolipoma was made by radiology. This could not explain the source of elevated serum cortisol since myelolipoma is seldom hormonally active.

Adrenal cortex scintigraphy with ${ }^{131}$ I-6beta-iodomethyl19-norcholesterol (NP-59) without dexamethasone premedication showed a unilateral tracer uptake (Fig. 1).

A laparoscopic adrenalectomy was performed. Histological examination confirmed the diagnosis of myelolipomatous adrenocortical adenoma, a rare but not uncommon condition (Fig. 1). The patient had significant clinical improvement postadrenalectomy.

\section{Discussion}

Some of the common adrenal neoplasm(s) encountered in clin- 


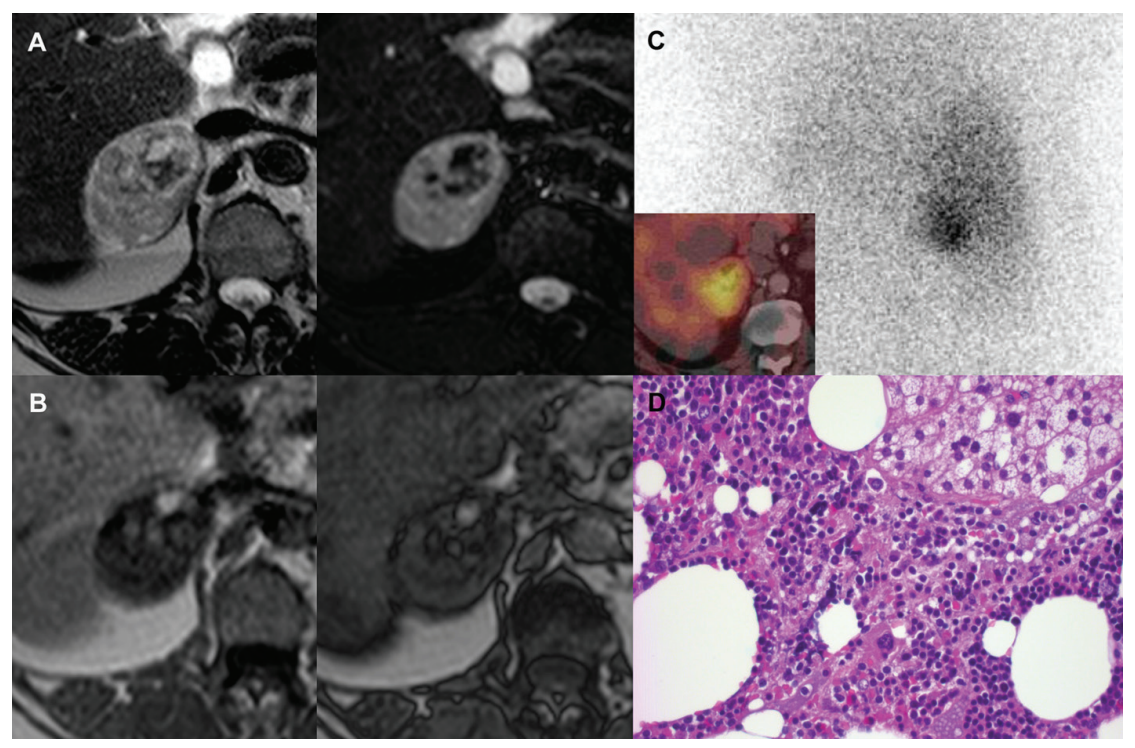

Figure 1. (A) Heterogenous signal on MRI. Fatty content areas with high T2 signal (upper left) and low T2 fat sat signal (upper right). (B) Absence of loss of signal intensity on out-of-phase T1 images (right image) compared to in-phase T1 images (left image), suggesting that there was no intracellular fat. (C) Unilateral uptake visible on ${ }^{131} \mathrm{I}$-19-iodocholesterol (planar static image, posterior view, SPECT and CT fusion image: small image) which was consistent with a cortisol-secreting adrenocortical tumor. (D) Islands of adrenal tumoral cells admixed with fatty and fibrous territories as well as hemorrhagic areas. Higher magnification showing details of the myeloid tissue with characteristic megakaryocytes (hematoxylin-eosin stain $\times 400$ ).

ical practice include benign adrenal adenoma, pheochromocytoma, myelolipoma, adrenal lymphomas and metastasis to adrenal glands from primary malignancies [1]. The imaging of the adrenal glands usually relies on lipid-sensitive techniques. An unenhanced CT of lipid rich tumors (which are mostly benign and non-functional) usually show a Hounsfield units (HFU) less than or equal to 10 while MRI shows signal loss on opposed-phase compared to in-phase T1-weighted images [1]. Positron emission tomography (PET) with fluorine-18 deoxyglucose (FDG) may sometimes be used to distinguish between benign (low PET uptake) from malignant adrenal mass (high PET uptake) [2].

Adrenal myelolipomas are usually benign and non-functioning masses that have adipose tissue and hematopoietic elements [3]. However, they may be indistinguishable from an adrenocortical adenoma with widespread myelolipomatous metaplasia through conventional imaging techniques like CT and MRI, and these tumors may be responsible for Cushing's syndrome [3]. In our case, adrenal scintigraphy was used to demonstrate an increased uptake in an otherwise benign looking adrenal myelolipoma on CT and MRI that turned out to be a functioning adrenal myelolipomatous adenoma.

There have been other reports in the literature describing the histopathology of the myelolipomatous adenomas: these lesions have sheets of adrenocortical cells with large areas of mature adipose tissue and infiltrating band of stromal small cells [4]. Rarely, extensive lipomatous metaplasia with myxoid change may be seen in an adrenocortical carcinoma [5].

On the other hand, rarely adreno myelolipoma may also cause increased uptake in FDG-PET suggesting some form of malignancy [6].

Adrenal scintigraphy with NP-59 (with concomitant
SPECT/CT) is an imaging technique that can help in distinguishing between aldosterone-producing adenoma (APA) and idiopathic adrenal hyperplasia (IAH) [7]. The technique is particularly suited if adrenal vein sampling (AVS) is inconclusive [7].

In a most recent retrospective chart review done at a single center, it was seen that adrenal cortical scintigraphy positivity was highly predictive in determining the outcome of subclinical Cushing's [8]. Adrenal scintigraphy had an accuracy of $93.7 \%$ for predicting post-operative hypoadrenalism in persons who had surgical resection [8]. In medically treated persons, a positive test was associated with worsening hyperglycemia and diastolic blood pressure [8].

\section{Conclusion}

Myelolipomatous adrenal adenoma (that may mimic adrenal myelolipoma on conventional imaging) may be a rare cause of subclinical Cushing's, and adrenal scintigraphy may be a useful imaging technique that may help in localizing the lesion, that can then be treated surgically.

\section{References}

1. Low G, Dhliwayo H, Lomas DJ. Adrenal neoplasms. Clin Radiol. 2012;67(10):988-1000.

2. Maurea S, Mainolfi C, Wang H, Varrella P, Panico MR, Klain M, Rossi R, et al. [Positron emission tomography (PET) with fludeoxyglucose F 18 in the study of adrenal 
masses: comparison of benign and malignant lesions]. Radiol Med. 1996;92(6):782-787.

3. Lamas C, Lopez LM, Lozano E, Atienzar M, RuizMondejar R, Alfaro JJ, Botella F. Myelolipomatous adrenal masses causing Cushing's syndrome. Exp Clin Endocrinol Diabetes. 2009;117(8):440-445.

4. Papotti M, Sapino A, Mazza E, Sandrucci S, Volante M, Bussolati G. Lipomatous Changes in Adrenocortical Adenomas: Report of Two Cases. Endocr Pathol. 1996;7(3):223-228.

5. Izumi M, Serizawa H, Iwaya K, Takeda K, Sasano H, Mukai K. A case of myxoid adrenocortical carcinoma with extensive lipomatous metaplasia. Arch Pathol Lab Med. 2003;127(2):227-230.
6. Castinetti F, Verschueren A, Cassagneau P, Brue T, Sebag F, Daniel L, Taieb D. Adrenal myelolipoma: an unusual cause of bilateral highly 18F-FDG-avid adrenal masses. J Clin Endocrinol Metab. 2012;97(8):2577-2578

7. Yen RF, Wu VC, Liu KL, Cheng MF, Wu YW, Chueh SC, Lin WC, et al. 131I-6beta-iodomethyl-19-norcholesterol SPECT/CT for primary aldosteronism patients with inconclusive adrenal venous sampling and CT results. J Nucl Med. 2009;50(10):1631-1637.

8. Ricciato MP, Di Donna V, Perotti G, Pontecorvi A, Bellantone R, Corsello SM. The role of adrenal scintigraphy in the diagnosis of subclinical Cushing's syndrome and the prediction of post-surgical hypoadrenalism. World J Surg. 2014;38(6):1328-1335. 